\title{
Axonics Sacral Neuromodulation System for Treating Refractory Overactive Bladder: A NICE Medical Technologies Guidance
}

\author{
Ruth Louise Poole ${ }^{1} \mathbb{D} \cdot$ Megan Dale $^{1} \mathbb{D} \cdot$ Helen Morgan $^{2}\left(\mathbb{D} \cdot\right.$ Tosin Oladapo $^{3} \mathbb{D} \cdot$ Rebecca Brookfield $^{3} \cdot$ Rhys Morris $^{1}(\mathbb{D}$
}

Accepted: 8 November 2021 / Published online: 29 December 2021

(c) The Author(s) 2021

\begin{abstract}
The Axonics sacral neuromodulation (SNM) system can be used by people with refractory overactive bladder (OAB) to reduce symptoms of urge urinary incontinence and urinary frequency, where conservative treatments have failed or are not suitable. It is the first system for this indication that makes use of a rechargeable battery to prolong the lifespan of the implanted device, with the potential advantage of reducing the frequency of surgical replacement procedures and associated complications. We describe the evidence considered by the UK National Institute of Health and Care Excellence (NICE) in their evaluation of this evidence, supported by Cedar Healthcare Technology Research Centre. Two observational studies provided descriptive data that suggested improvement in control of symptoms after implantation of the Axonics SNM system; however, there was no peer-reviewed evidence that directly compared rechargeable and non-rechargeable SNM systems. In the absence of long-term data, economic modelling relies on the accuracy of battery life estimates. The evidence supports the case for adopting the Axonics SNM system for treating refractory $\mathrm{OAB}$, when conservative treatment or treatment with medicines has not worked. This conclusion is consistent with other relevant NICE guidelines. Use of Axonics SNM technology in the UK National Health Service (NHS) is associated with a potential cost saving of $£ 6025$ per person over a 15 -year period when compared with an equivalent non-rechargeable SNM system, assuming the claimed battery life estimate (a minimum of 15 years) is accurate. The cost savings are estimated to start around 6 years after implantation.
\end{abstract}

Ruth Louise Poole

Ruth.Poole@wales.nhs.uk

Megan Dale

Megan.Dale@wales.nhs.uk

Helen Morgan

MorganHE1@cardiff.ac.uk

Tosin Oladapo

Tosin.Oladapo@nice.org.uk

Rebecca Brookfield

Rebecca.Brookfield@nice.org.uk

Rhys Morris

Rhys.Morris@wales.nhs.uk

1 Cedar, Cardiff and Vale University Health Board, Cardiff, UK

2 Cedar, Cardiff University, Cardiff, UK

3 National Institute for Health and Care Excellence, Manchester, UK

\section{Key Points for Decision Makers}

Published evidence from two non-comparative observational studies suggests that use of the Axonics Sacral Neuromodulation (SNM) system can help people with overactive bladder (OAB) to manage symptoms of urinary frequency and urinary incontinence.

National Institute of Health and Care Excellence (NICE) concluded that the Axonics SNM system should be considered as an option for people with refractory OAB when conservative treatment or treatment with medicine has not worked.

If the device battery remains functional for 15 years, the Axonics SNM system is estimated to save the National Health Service (NHS) around $£ 6025$ per person, when compared with a non-rechargeable system; however, at present there are no long-term data that directly compare both types of systems. 


\section{Introduction}

Medical technologies guidance (MTG) $[1,2]$ is produced by National Institute of Health and Care Excellence (NICE) as part of the Medical Technologies Evaluation Programme (MTEP). It is intended to facilitate adoption of new or innovative medical devices or diagnostics in the UK National Health Service (NHS) where supported by evidence of clinical effectiveness and economic evidence showing a potential for cost savings compared with standard care. This article describes the development of guidance and recommendations about the Axonics sacral neuromodulation (SNM) system for the treatment of refractory overactive bladder (OAB). Other papers in this series report similar guidance development processes for a range of innovative medical technologies.

The initial assessment report [3] was produced by Cedar Healthcare Technology Research Centre, an NHS academic evaluation centre that is part of Cardiff and Vale University Local Health Board and Cardiff University. The assessment report informed the deliberations of NICE's Medical Technologies Appraisal Committee (MTAC) and the subsequent MTG recommendations for adoption and use of the Axonics SNM system in the NHS [4].

\subsection{Background to Technology and Application}

SNM can be used to control symptoms of OAB such as urinary frequency and urge urinary incontinence (urinary leaks), if medicines or other treatments are not suitable or have not worked. The Axonics SNM system is also intended to treat symptoms of urinary retention and chronic faecal incontinence, but the scope of this evaluation only considered the treatment of symptoms of OAB.

The Axonics SNM system is designed to stimulate the sacral nerve to improve bladder control. Lead electrodes transmit a pulse from an implantable pulse generator (stimulator) to the sacral nerve. The stimulator is surgically implanted beneath the skin of the upper buttock. It is powered by a battery that is recharged every $1-2$ weeks using a wireless charger attached to the skin. At the time of submission, the main innovative aspect of the Axonics SNM system was the rechargeable battery. The battery was expected to function for at least 15 years, which could reduce the frequency of surgical replacement when compared with non-rechargeable SNM systems (that are explanted and replaced every $4-5$ years). Other benefits claimed by the company were magnetic resonance imaging (MRI) compatibility and a smaller device size-considered to be more comfortable for people with a low body mass index (BMI).

In usual clinical practice, devices are temporarily implanted to confirm the response to stimulation before a second procedure in which the stimulator is permanently implanted. When reporting study outcomes, 'test responders' refers to the initial test of the device function, and 'therapy responders' refers to the therapeutic response in reducing symptoms of OAB.

\subsection{Decision Problem (Scope)}

In their evidence submission, the company must keep within the scope of the evaluation or provide a rationale for any variance. The scope is defined by NICE in the form of a PICO table (population, intervention, comparator, outcomes), including cost analysis and subgroups to be considered.

\subsection{Population}

The population included people with symptoms of OAB for whom conservative therapy and drug treatment have failed or are not suitable. A subgroup for particular consideration was described as slim people with lower than average BMI and a paucity of subcutaneous buttock fat.

\subsection{Intervention}

The intervention was defined as the Axonics SNM system.

\subsection{Comparator}

The comparator was defined as other SNM systems.

\subsection{Outcomes}

The following outcomes were included in the scope: responder rate (percentage of patients who experience 50\% or more reduction in their leaks compared with baseline); level of reduction in OAB symptoms (such as average daily number of urgency leaks); number of surgical interventions to replace SNM devices and the risks associated with these procedures; time to battery depletion; ease of use of device; procedure-related infection rates; incidence of therapeutic failure; change in quality of life, including pain and discomfort; explantation rate due to MRI; time to revision surgery; level of patient and carer satisfaction; and device-related adverse events (AEs). 


\section{Cedar's Review of the Evidence}

The company, Axonics Inc., provided an evidence submission to NICE, presenting the available clinical and cost evidence alongside a de novo cost model. Cedar's assessment report aimed to provide the NICE MTAC with a balanced, fair and independent appraisal of the evidence surrounding use of the Axonics SNM system for the treatment of refractory $\mathrm{OAB}$.

\subsection{Review of Clinical Effectiveness Evidence}

The company submitted evidence based on 13 articles, 6 relating to the Axonics SNM system (4 published, 2 unpublished) and 7 relating to a non-rechargeable SNM system (Interstim, Medtronic). None of the studies provided data that directly compared the clinical effectiveness of the Axonics SNM system with an alternative SNM system.

To confirm that all key studies had been identified, Cedar ran a comprehensive search across 10 databases and 2 clinical trial registries, using a range of free text terms and subject headings. Studies that did not use Axonics SNM in either the treatment or comparator arm were excluded. Cedar concluded that 6 papers relating the Axonics system were relevant to the decision problem, all of which had been identified by the company.

All relevant articles were based on two clinical studies, each series reporting outcomes after increasing lengths of follow-up. The ARTISAN-SNM [5-7] and the RELAXOAB [8-10] studies are summarised in Tables 1 and 2. A further paper about device programming settings for RELAX-OAB provided additional detail [11]. Subsequent to the initial literature search, new peer-reviewed publications $[12,13]$ verified the content of the previously unpublished papers.

Both trials are before and after, intrapatient, observational studies reporting patient outcomes as a change from baseline. Neither followed the usual practice of a twostage procedure, in which a test of device functioning is carried out before permanent implantation. In both studies, the devices were permanently implanted in a single procedure; the researchers considered the initial 2-4 weeks of implantation to be equivalent to the test phase.

One of the key innovative features of the Axonics SNM system was considered to be the incorporation of a rechargeable battery into the implantable pulse generator device. The company's value proposition was based on the battery having a lifespan of at least 15 years, but clinical effectiveness had not been demonstrated for longer than 2 years. NICE commissioned a separate technical assessment, which concluded "it seems likely that for typical stimulus parameters and for the manufacturer-recommended 1-h-per-week recharge regime, a claimed battery life of 15 years is defensible" [14].

\subsection{Safety Outcomes}

Numbers of adverse events (AEs) reported by the two clinical studies are summarised in Tables 1 and 2. No serious device- or procedure-related AEs were reported and there were no unanticipated AEs in either study. All incidents of discomfort associated with stimulation were resolved with reprogramming.

Including test non-responders, devices were explanted from 4 of 129 people (3\%) after 1 year (ARTISAN-SNM) and from 7 of 51 people after 2 years (14\%) (RELAX-OAB). One lead migration was reported within the first 6 months of each study, both of which resolved as a result of lead revision procedures.

Clinical experts, consulted as part of the NICE process, speculated that long-term implantation may be associated with an increased risk of pain as a result of lead migration or stimulator movement, or that there may be issues with longterm implantation of a lithium device. Conversely, there is anecdotal evidence that the smaller size of the Axonics stimulator is associated with a reduced risk of pain and discomfort when compared with larger non-rechargeable devices. Experts with experience using other SNM systems believe that tolerability is likely to be sustained in the longer term.

The company's submission observes that adverse events are not expected to differ substantially between SNM systems, which was also confirmed by expert advisers. A possible exception is that the long-term incidence of surgical complications could be reduced with rechargeable systems, as battery replacement procedures are anticipated to be required less frequently.

\subsection{Review of Economic Evidence}

The company submitted 19 studies, however none of these met the requirement of the scope. Cedar did not identify any economic evidence directly concerning Axonics. Two studies contained information relevant to the economic model. Freemantle et al. [15] reported costs that informed the supportive care arm, while Noblett et al. [16] reported an economic model for an alternative SNM device, in a US setting, and this was used as a basis for the model submitted by the company.

\subsubsection{Axonics Model Structure}

The submitted model was a Markov model with a 3-month cycle, based on an NHS and personal social services 


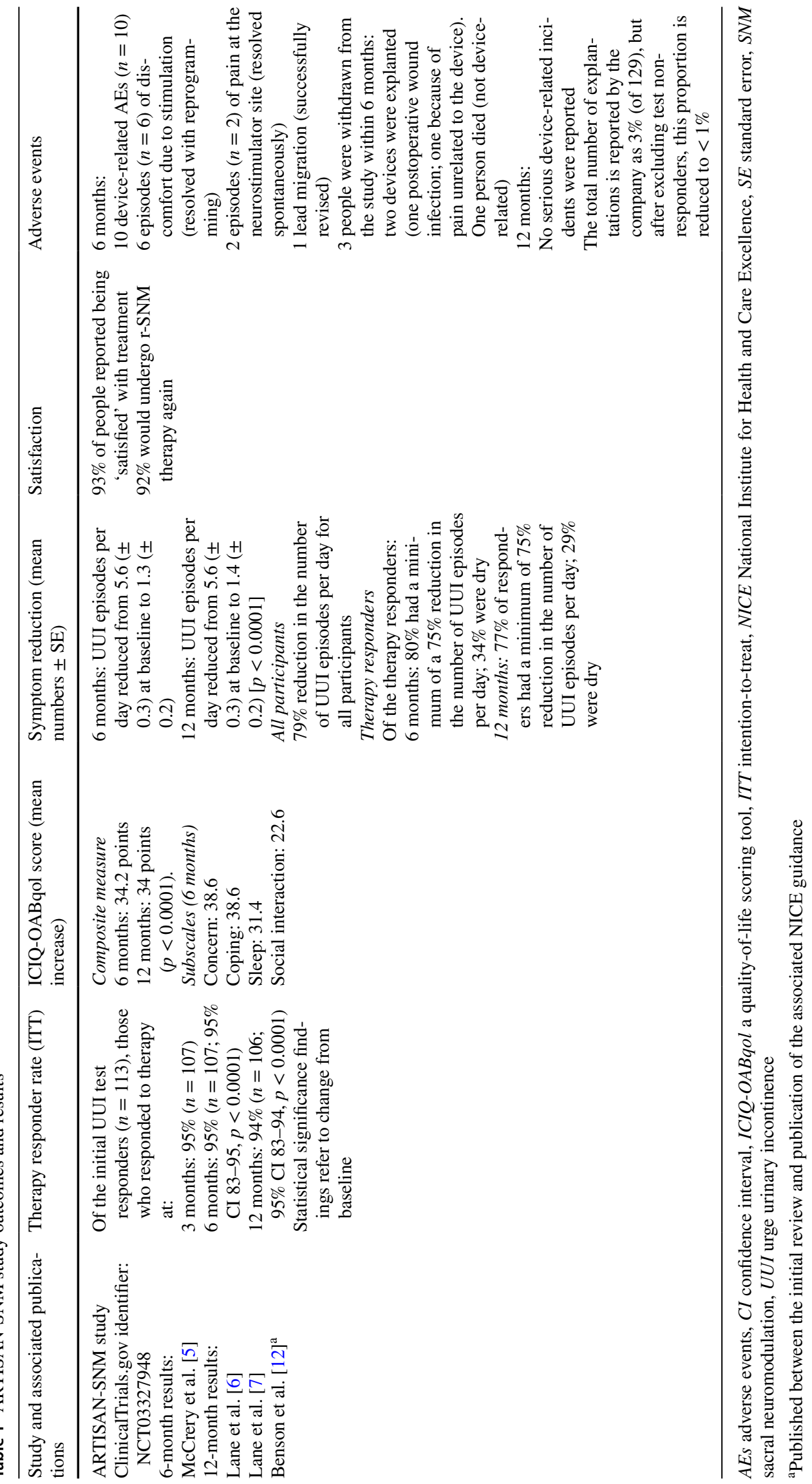




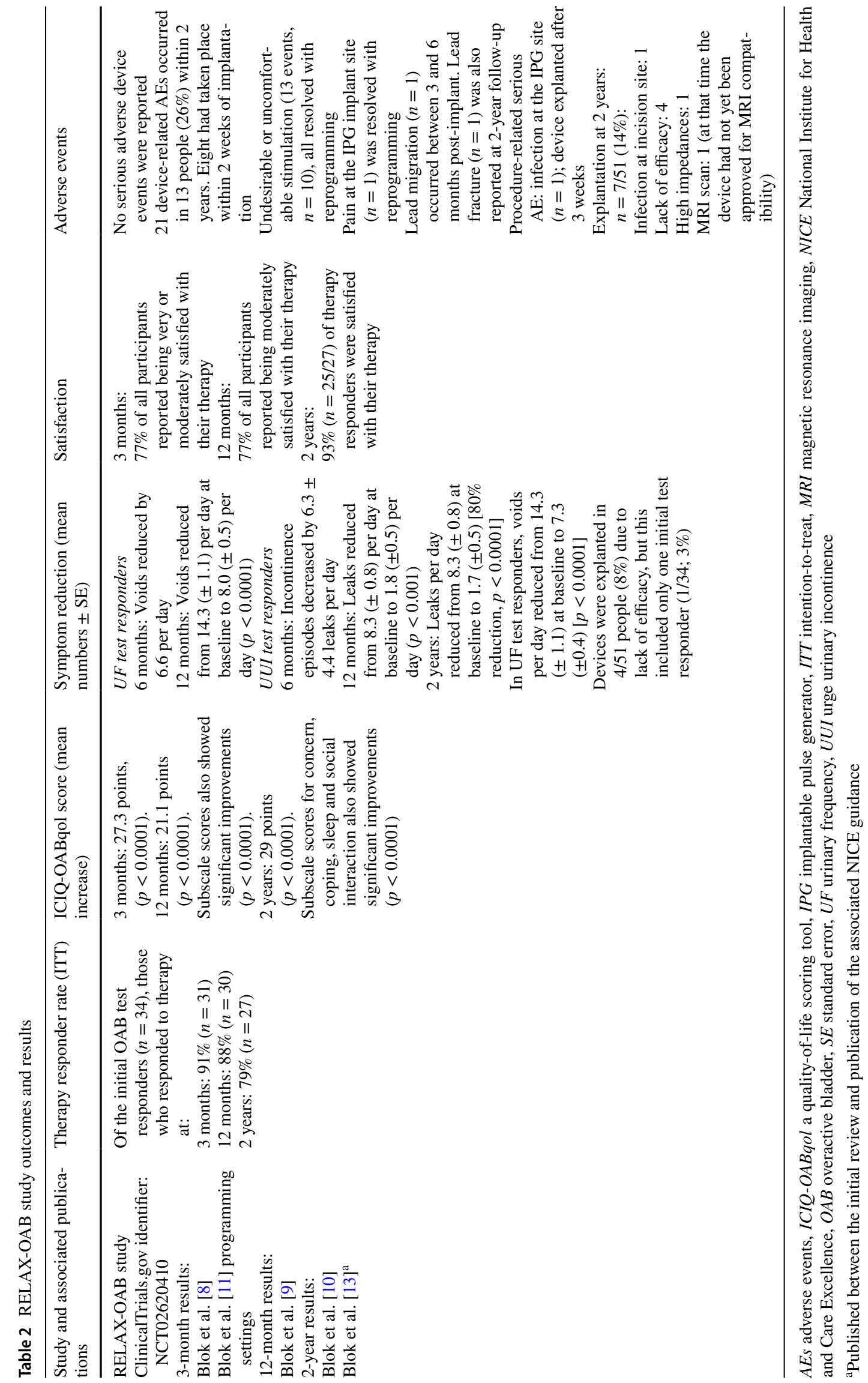


perspective. It had a 15-year time horizon to match the intended battery life of the rechargeable device. An annual discount rate of $3.5 \%$ per annum for costs arising after the first year was applied (as per the NICE MTEP methods guide). The model compared two SNM systems: rechargeable Axonics (Axonics Modulation Technologies, Inc.) and a non-rechargeable system (InterStim, Medtronic, Inc.).

The modelled health states were: on SNM therapy, off SNM therapy, and dead. SNM therapy was either rechargeable or non-rechargeable, depending on the arm of the model. Patients were considered to move between states based on transition properties for mortality and discontinuation of therapy.

\subsubsection{Key Assumptions}

The key assumptions in the company model, and actions taken by Cedar to address them, were:

- There are no differences in SNM therapy effectiveness, discontinuation or adverse event rates between rechargeable and non-rechargeable systems (changes were made by Cedar using rates specific to each device type for the accepted model).

- Differences in the rechargeable and non-rechargeable device battery lifetimes lead to a reduced need for rechargeable device replacements, and thus a reduction in procedure-related adverse events.

- There are no differences in testing procedures and test outcomes prior to full implantation of an SNM device, therefore this was not included in the model (this was included in an additional scenario).

- Adverse events are assumed to happen in the same cycle as the procedure (Cedar carried out additional work to allow lead migration, breakage and pain to continue throughout the model).

- The likelihood of infection or pain is reduced at subsequent procedures (a conservative assumption as it reduces the cost associated with replacements).

- Procedures are a 1-day inpatient admission rather than day case (this favoured the Axonics SNM intervention and was changed by Cedar following consultation with clinical experts).

- Efficacy is $100 \%$ for patients who continue therapy. Incontinence costs are incurred only for patients who discontinue therapy.

\subsubsection{Data Sources for Outcomes and Resources}

Clinical parameters were derived from the InSite study [17], which were also used for the economic evaluation of rechargeable and non-rechargeable devices in the US setting [16]. Discontinuation rates for the first year were taken from
Noblett et al. [17], with subsequent years taken from Chughtai et al. [18] for both rechargeable and non-rechargeable devices.

Data for the adverse events of surgical pain, lead migration and lead fracture were taken from the InSite study [17], with additional information from the Principal Investigator of the InSite study. Surgical site infection was also included as an adverse event and was modelled as being treated by intravenous antibiotics with an inpatient stay, or replacement of the entire implantable device.

Values for gender distribution, InterStim technology lifetime, and frequency of programming visits are derived from other sources $[15,19,20]$.

Resource use was based on the direct cost of implanting and management of rechargeable and non-rechargeable systems, as well as associated adverse events. The technology would not change any aspects of the patient pathway or current practice and was assumed to be as effective as current SNM systems. The company did not identify any extra resource use associated with implementation in the NHS. All procedures have a cost to the NHS [21], with an additional cost for each device component. The technology costs (device and accessories; excluding value-added tax [VAT]) of both rechargeable and non-rechargeable systems were obtained from the NHS Supply Chain [22].

The key parameters were the expected lifetime of the Axonics implantable device at 15 years, and the lifetime of a non-rechargeable implantable device at 4.4 years.

\subsubsection{Changes by Cedar}

Cedar made minor corrections to calculations, including the inflation index, cumulative discontinuation of therapy, VAT adjustment, and the cost of the comparator trial stimulator. The overall impact was an increase in cost saving of the Axonics SNM system compared with the non-rechargeable system.

Cedar also made a number of changes to the inputs and structure of the model. For the discontinuation rate of the non-rechargeable system, Cedar interpreted the available data [18] to give a 5-year discontinuation rate of $17 \%$ rather than $6 \%$. Cedar also changed the clinical inputs for rechargeable systems, to be based on studies using the rechargeable system [8-11, 13] rather than data from the Insite study [17]. The probability of lead migration or fracture was changed to be constant throughout the model, meaning it was no longer occurring only in the cycle with an implantation or revision procedure. In addition, initial implantation costs were changed from elective inpatient to day-case procedures.

After consultation, Cedar researchers were asked to consider additional changes to the model. Expert advice suggested that rates of surgical site infection were low for both systems, and the Committee considered it appropriate to use 


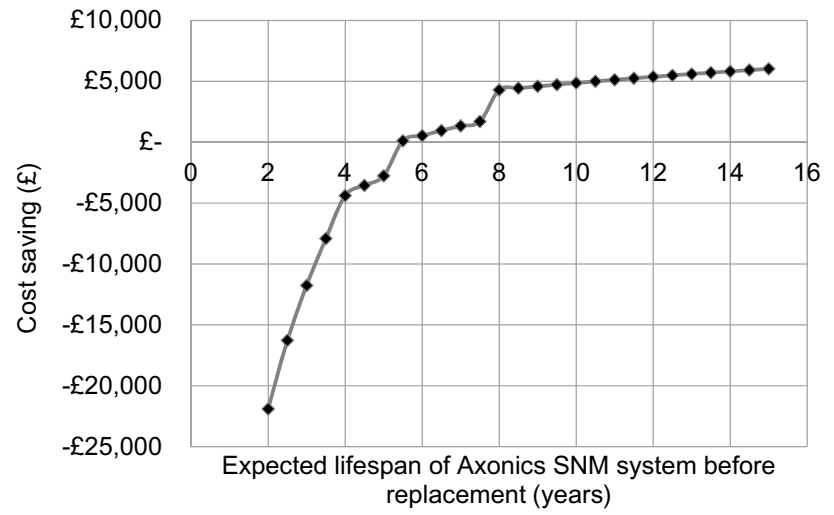

Fig. 1 Threshold diagram showing the impact of the Axonics SNM device lifespan on cost saving

the same rate of $1 \%$ for both technologies. This resulted in a small reduction in the cost saving.

\subsubsection{Results}

The model submitted by the company found a cost saving of $£ 6038$, while Cedar's base-case considered by the Committee found a cost saving of $£ 6345$. Changes after consultation resulted in a reduced cost saving of $£ 6025$ for the Axonics SNM system at 15 years compared with a non-rechargeable system.

\subsubsection{Sensitivity Analysis}

Cedar conducted one-way sensitivity analysis, modelled additional scenarios, and extended the time horizons. The key driver in the model was the lifespan of the implantable device before replacement, therefore a threshold diagram for the time-to-replacement of the rechargeable device (Fig. 1) was created. The impact of variation in time-to-replacement for both devices was investigated using a two-way analysis (Table 3).

\section{National Institute for Health and Care Excellence Guidance}

\subsection{Development of Guidance}

NICE's MTAC met in January 2020 and considered evidence from a range of sources, including the company's submission, Cedar's assessment report [3], a technical assessment on battery life expectancy [14], a patient organisation, a patient survey, and testimony from clinical experts. Provisional recommendations were subject to public consultation between 14 February 2020 and 13 March 2020. NICE received 19 comments from four consultees (representing the company, a comparator company, and two professional societies). Comments covered issues such as new comparator SNM systems, MRI scan compatibility, draft recommendations, and wording changes.

\subsection{Recommendations}

At a second MTAC meeting in June 2020, the Committee considered the responses to public consultation as well as additional work carried out by the external assessment centre. The committee then made the following recommendations:

- Evidence supports the case for adopting the SNM system for treating refractory OAB in the NHS. The Axonics SNM system improves symptoms and quality of life, and

Table 3 Impact on incremental cost saving for the Axonics rechargeable SNM compared with a non-rechargeable system, considering variation in time-to-replacement for rechargeable and non-rechargeable SNM systems

\begin{tabular}{|c|c|c|c|c|c|c|c|}
\hline & \multicolumn{7}{|c|}{ Expected lifetime for the Axonics rechargeable system (years) } \\
\hline & 2 & 5 & 7 & 9 & 11 & 13 & 15 \\
\hline \multicolumn{8}{|c|}{ Expected lifetime of comparator (years) } \\
\hline 2.0 & $-£ 5447$ & $£ 13,692$ & $£ 17,785$ & $£ 21,034$ & $£ 21,579$ & $£ 22,055$ & $£ 22,472$ \\
\hline 3.0 & $-£ 14,556$ & $£ 4583$ & $£ 8677$ & $£ 11,926$ & $£ 12,471$ & $£ 12,946$ & $£ 13,363$ \\
\hline 4.0 & $-£ 21,186$ & $-£ 2047$ & $£ 2046$ & $£ 5296$ & $£ 5841$ & $£ 6316$ & $£ 6733$ \\
\hline 4.4 & $-£ 21,894$ & $-£ 2755$ & $£ 1338$ & $£ 4588$ & $£ 5133$ & $£ 5608$ & $£ 6025$ \\
\hline 5.0 & $-£ 22,648$ & $-£ 3509$ & $£ 584$ & $£ 3834$ & $£ 4379$ & $£ 4854$ & $£ 5271$ \\
\hline 6.0 & $-£ 25,616$ & - £6477 & $-£ 2383$ & $£ 866$ & $£ 1411$ & $£ 1886$ & $£ 2303$ \\
\hline 7.0 & $-£ 26,327$ & $-£ 7188$ & $-£ 3094$ & $£ 155$ & $£ 700$ & $£ 1175$ & $£ 1592$ \\
\hline
\end{tabular}

EAC base case result, incremental, with 15 years expected lifespan for Axonics SNM device and 4.4 years expected lifespan for the comparator (in bold) 
also has a longer battery life than the non-rechargeable system used in NHS clinical practice.

- The Axonics SNM system should be considered as an option for people with refractory OAB; that is, when conservative treatment or treatment with medicine has not worked, in line with NICE's guidelines on urinary incontinence and pelvic organ prolapse [23] and lower urinary tract symptoms [24]. The Axonics SNM system is small and does not need to be removed for most types of MRI scans, therefore it may be useful for people with a low BMI or when an MRI is likely.

- Cost modelling estimates that, over 15 years, the Axonics SNM system is cost saving, compared with the nonrechargeable system, by about $£ 6025$ per person. Cost savings are estimated to begin 6 years after implant. This is because the device needs to be replaced less frequently than the non-rechargeable system, assuming Axonics has a lifespan of at least 15 years. For more details, see the NICE resource impact statement [25].

\section{Key Challenges and Learning Points}

All papers relevant to assessment of the Axonics system are derived from two observational, single-arm studies. The RELAX-OAB and ARTISAN-SNM studies add weight to the existing body of evidence that SNM devices are a useful treatment option in people with refractory OAB. When people compared their symptoms before and after implantation of the Axonics SNM system, average episodes of urge incontinence and urinary frequency had reduced, with corresponding improvements in quality of life. Because of a lack of statistically comparative data between the Axonics device and a non-rechargeable comparator, we were unable to conclusively verify claims of equivalent clinical effectiveness. The main value proposition of rechargeable systems relies on a long battery life, but, in the absence of long-term data, we could not confirm any potential clinical advantages of this technology over non-rechargeable SNM systems.

Similarly, the economic model relies on the accuracy of the predicted lifetime of the rechargeable device. As the battery has a long expected lifetime (15 years), the model inputs could not be based on real-world data. Instead, we carried out sensitivity analyses to evaluate the impact of any variations in time-to-replacement estimates.

\section{Conclusions}

NICE assessed the Axonics SNM system for treating refractory OAB to help the NHS decide whether to use this product. Assuming the lifespan of the battery exceeds 15 years, cost modelling indicates that the Axonics SNM system could save the NHS around $£ 6025$ per person over 15 years when used as an alternative to a non-rechargeable system. Cost savings are estimated to begin 6 years after device implantation.

Therefore, NICE concluded that the Axonics SNM system should be considered as an option for people with refractory $\mathrm{OAB}$ when conservative treatment or treatment with medicine has not worked.

\section{Declarations}

Author contributions RLP, MD, HM, RB, and TO contributed to the preparation of this manuscript. RM reviewed the article, and can act as a guarantor for the overall content.

Compliance with ethical standards RLP, MD and RM are NHS employees, which has a financial interest in the guidance on which this project is based. HM is a Cardiff University employee and has no conflicts of interest to declare. RB and TO are NICE employees and had no role in the production of the assessment report but contributed to the preparation of this manuscript. This summary of the Medical Technology Guidance was produced following publication of the final guidance report. The article has not been externally peer reviewed by Applied Health Economics and Health Policy but has been reviewed externally by NICE. Cedar was funded by the NICE MTEP for their work.

Funding National Institute for Health and Care Excellence (External Assessment Centre contract).

Open Access This article is licensed under a Creative Commons Attribution-NonCommercial 4.0 International License, which permits any non-commercial use, sharing, adaptation, distribution and reproduction in any medium or format, as long as you give appropriate credit to the original author(s) and the source, provide a link to the Creative Commons licence, and indicate if changes were made. The images or other third party material in this article are included in the article's Creative Commons licence, unless indicated otherwise in a credit line to the material. If material is not included in the article's Creative Commons licence and your intended use is not permitted by statutory regulation or exceeds the permitted use, you will need to obtain permission directly from the copyright holder. To view a copy of this licence, visit $\mathrm{http} / / /$ creativecommons.org/licenses/by-nc/4.0/.

\section{References}

1. Campbell B, Campbell M. NICE medical technologies guidance: a novel and rigorous methodology to address a new health technology assessment challenge. Appl Health Econ Health Policy. 2012;10(5):295-7.

2. National Institute for Health and Care Excellence. Medical technologies guidance. London: NICE; 2021. Available at: https:// www.nice.org.uk/About/What-we-do/Our-Programmes/NICEguidance/NICE-medical-technologies-guidance. Accessed 14 May 2021.

3. Poole RL, Dale M, Morgan H, Ryczek E, Cohen B, Carolan-Rees G. Axonics sacral neuromodulation system for treating refractory 
overactive bladder (external assessment report). 2019. Available at: https://www.nice.org.uk/guidance/mtg50/documents/suppo rting-documentation-2. Accessed 21 Mar 2021.

4. National Institute for Health and Care Excellence. Axonics sacral neuromodulation system for treating refractory overactive bladder. London: NICE; Sep 2020. (Medical technologies guidance [MTG50]). Available at: https://www.nice.org.uk/guidance/ mtg50. Accessed 21 Mar 2021.

5. McCrery R, Lane F, Benson K, et al. Treatment of urinary urgency incontinence using a rechargeable SNM system: 6-month results of the ARTISAN-SNM study. J Urol. 2020;203:185-92.

6. Lane F, McCrery R, Taylor C, et al. Treatment of urinary urgency incontinence with the Axonics miniaturised, rechargeable SNM system: clinical outcomes of the ARTISAN-SNM pivotal study. Int Urogynecol J. 2019;30:1-29. https://doi.org/10.1007/s00192019-04123-4. In: AUGS/IUGA Scientific Meeting: Abstracts of Long Oral Presentations, IUGA 44th Annual Meeting-Nashville, TN, USA, September 24-28, 2019.

7. Benson K, McCrery R, Taylor C, et al. One-year outcomes of the Axonics system for treatment of urinary urgency incontinence. SUFU 2020 Winter Meeting abstract. Podium \#29: Available at: https://sufuorg.com/docs/meetings/sufu2002/2020-program-bookabstracts.aspx. Accessed 21 Mar 2021. (An early draft of this abstract was submitted by the company to NICE, with Lane listed as the first author).

8. Blok B, Van Kerrebroeck P, de Wachter S, et al. Three month clinical results with a rechargeable sacral neuromodulation system for the treatment of overactive bladder. Neurourol Urodyn. 2018;37(S2):S9-16.

9. Blok B, Van Kerrebroeck P, de Wachter S, et al. A prospective, multicenter study of a novel, miniaturized rechargeable sacral neuromodulation system: 12-month results from the RELAX-OAB study. Neurourol Urodyn. 2019;38(2):689-95.

10. Blok B, de Wachter S, Ruffion A et al. Two years outcomes of the treatment of overactive bladder with the miniaturized, rechargeable Axonics system. International Continence Society. 2019 [abstract and e-poster]. Available at: https://www.ics.org/2019/ abstract/158. Accessed 21 Mar 2021.

11. Blok B, Van Kerrebroeck P, de Wachter S, et al. Programming settings and recharge interval in a prospective study of a rechargeable sacral neuromodulation system for the treatment of overactive bladder. Neurourol Urodyn. 2018;37(Suppl 2):S17-22.

12. Benson $\mathrm{K}$, McCrery R, Taylor $\mathrm{C}$, et al. One-year outcomes of the ARTISAN-SNM study with the Axonics system for the treatment of urinary urgency incontinence. Neurourol Urodyn. 2020;39:1482-8.

13. Blok B, Van Kerrebroeck P, de Wachter S, et al. Two-year safety and efficacy outcomes for the treatment of overactive bladder using a long-lived rechargeable sacral neuromodulation system. Neurourol Urodyn. 2020;39:1108-14.
14. Bangel KA, Cole H, Drinnan M. MT417 Axonics sacral neuromodulation system for bladder control in people with symptoms of overactive bladder: external assessment centre technical assessment report. Newcastle: EAC; 2020. Available at: https://www. nice.org.uk/guidance/mtg50/documents/supporting-documentat ion-2. Accessed 21 Mar 2021.

15. Freemantle N, Khalaf K, Loveman C, et al. Onabotuliniumtoxin A in the treatment of overactive bladder: a cost-effectiveness analysis versus best supportive care in England and Wales. Eur J Health Econ. 2016;17:911-21.

16. Noblett KL, Dmochowski RR, Vasavada SP, Garner AM, Liu $\mathrm{S}$, Pietzsch JB. Cost profiles and budget impact of rechargeable sacral neuromodulation devices in the treatment of overactive bladder syndrome. Neurourol Urodyn. 2017;36:727-33.

17. Noblett K, Siegel S, Mangel J, et al. Results of a prospective, multicenter study evaluating quality of life, safety, and efficacy of sacral neuromodulation at twelve months in subjects with symptoms of overactive bladder. Neurourol Urodyn. 2016;35:246-51.

18. Chughtai B, Sedrakyan A, Issacs A, Lee R, Te A, Kaplan S. Long term safety of sacral nerve modulation in Medicare beneficiaries. Neurourol Urodyn. 2015;34:659-63.

19. Cameron AP, Anger JT, Madison R, et al. Battery explantation after sacral neuromodulation in the Medicare population. Neurourol Urodyn. 2013;32:238-41.

20. Suskind AM, Dunn RL, Kaufman SR, et al. Understanding the dissemination of sacral neuromodulation. Surg Innov. 2013;20:625-30.

21. NHS Improvement. National Schedule of Reference Costs 2017/18. Available at: https://webarchive.nationalarchives.gov. uk/20200501111106/https://improvement.nhs.uk/resources/refer ence-costs/. Accessed 21 Mar 2021

22. NHS Supply Chain. Available at: https://www.supplychain.nhs. uk/. Accessed Sep 2019.

23. National Institute for Health and Care Excellence. Urinary incontinence and pelvic organ prolapse in women: management. London: NICE; Apr 2019, updated June 2019 (NICE guideline [NG123]). Available at: https://www.nice.org.uk/guidance/ng123. Accessed 21 Mar 2021.

24. National Institute for Health and Care Excellence. Lower urinary tract symptoms in men: management. London: NICE; May 2010, updated June 2015 (Clinical guideline [CG97]). Available at: https://www.nice.org.uk/guidance/cg97. Accessed 21 Mar 2021.

25. National Institute for Health and Care Excellence. Axonics sacral neuromodulation system for treating refractory overactive bladder: resource impact statement. Available at: https://www.nice.org. uk/guidance/mtg50/resources/resource-impact-statement-88383 83005. Accessed 21 Mar 2021. 